\title{
On A Lower Bound For The Rank Of A Partitioned Square Matrix
}

\author{
By Gene H. Golub
}

It has been shown by Fan and Hoffman [1] that it is possible to find lower bounds for the rank of a square matrix that can be calculated in a simple fashion from the coefficients of the matrix. In this note, we shall extend their results to matrices which have been partitioned into blocks.

Throughout this note, we shall use the following notation:

$$
\begin{aligned}
r(A) & =\operatorname{rank} \text { of } A ; \\
\operatorname{tr}(A) & =\text { trace of } A ; \\
|A| & =\left(\left|a_{i j}\right|\right) ; \\
\lambda_{i}(A) & \text { are the eigenvalues of } A, \text { and } \\
\rho(A) & =\max _{i}\left|\lambda_{i}(A)\right| .
\end{aligned}
$$

Let $A$ be an arbitrary $n \times n$ complex matrix partitioned as follows:

$$
A=\left(\begin{array}{cccc}
A_{1,1} & A_{1,2} & \cdots & A_{1, N} \\
A_{2,1} & A_{2,2} & \cdots & A_{2, N} \\
: & : & & \\
A_{N, 1} & A_{N, 2} & \cdots & A_{N, N}
\end{array}\right)
$$

with $A_{i j}$ an $n_{i} \times n_{j}$ matrix so that the diagonal matrices are square. The diagonal elements of $A_{m m}$ are denoted as $a_{k k}^{(m)}, k=1, \cdots, n_{i} ; m=1,2, \cdots, N$.

Associated with each block $A_{i j}$ is a norm, $\left\|A_{i j}\right\|$, which has the usual properties [3], and which is compatible with a vector norm, i.e.,

$$
\left\|A_{i j} x\right\| \leqq\left\|A_{i j}\right\| \cdot\|x\| \text {. }
$$

In addition, we require that the matrix norm have the following property:

If $D$ is a diagonal matrix whose entries are equal to one in modulus, then

$$
\|D A\|=\|A\| \text {. }
$$

This property is satisfied, for example, by the spectral norm and those norms dependent only on the absolute value of the elements, e.g., the euclidean norm.

In order to obtain a lower bound for $r(A)$, it is necessary to obtain an upper bound for $\rho(A)$. To this end, we have

TheOREM 1:

$$
\rho(A) \leqq \max _{1 \leqq i \leqq N} \sum_{j=1}^{N}\left\|A_{i, j}\right\|
$$

Received July 9, 1962. 
Proof:

Since $A x=\rho(A) x$,

$$
\rho(A)\left\|x_{i}\right\| \leqq \sum_{j=1}^{n}\left\|A_{i, j}\right\|\left\|x_{j}\right\| \quad \text { for } \quad i=1,2, \cdots, N .
$$

Thus if $\left\|x_{I}\right\| \geqq\left\|x_{i}\right\|$ for all $i$,

$$
\rho(A) \leqq \sum_{n=1}^{N}\left\|A_{I, j}\right\| \cdot \frac{\left\|x_{j}\right\|}{\left\|x_{I}\right\|} \leqq \max _{1 \leqq i \leqq N} \sum_{j=1}^{N}\left\|A_{i, j}\right\| .
$$

Theorem 1 is a generalization of the Gerschgorin theorem and can also be derived from the generalization of the Gerschgorin Circle Theorem by Feingold and Varga [2].

Theorem 2. For any partitioned matrix $A$,

$$
r(A) \geqq \sum_{i=1}^{N} \frac{\operatorname{tr}\left|A_{i, i}\right|}{\sum_{j=1}^{N}\left\|A_{i, j}\right\|}
$$

(Whenever $0 / 0$ occurs on the r.h.s., we agree to put $0 / 0=0$.)

Proof:

Let

$$
\begin{aligned}
& b^{(m)}=\sum_{j=1}^{n}\left\|A_{m, j}\right\| \quad m=1,2, \cdots N, \\
& a_{k k}^{(m)}=\rho_{k m} e^{i \theta_{k m}}
\end{aligned}
$$

and let

$$
C=E A
$$

where $E$ is a diagonal matrix with

$$
\begin{aligned}
& e_{k k}^{(m)}=\frac{e^{-i \theta_{k m}}}{b^{(m)}} \quad \text { for } \quad b^{(m)} \neq 0 \\
& =1 \text { for } b^{(m)}=0
\end{aligned}
$$

$\left(k=1, \cdots, n_{i} ; m=1, \cdots, N\right)$.

Since $\operatorname{det} C \neq 0, \quad r(C)=r(A)$.

Now $\max _{1 \leqq i \leqq N} \sum_{j}\left\|C_{i j}\right\|=1$, so $\rho(C) \leqq 1$ by Theorem 1 .

Since $\operatorname{tr}(C)$ is equal to the sum of the non-zero eigenvalues, and since $\rho(C) \leqq$ 1 , it follows that

$$
\operatorname{tr}(C) \leqq r(C)
$$

It is obvious that the bound for $r(A)$ depends very much upon the partitioning of the matrix $A$ and the norm used. Indeed, it is not difficult to construct examples where if $n_{i} \geqq 1$, then the bound for $r(A)$ may be inferior to that when $n_{i}=1$ for all $i$. The question arises whether it is possible to choose partitionings and norms to maximize the lower bound for $r(A)$.

Space Technology Laboratories, Inc.

One Space Park

Redondo Beach, California 
1. KY Fan \& A. J. Hofrman, "Lower bounds for the rank and location of the eigenvalues of a matrix," Contributions to the Solution of Systems of Linear Equations and the Determination of Eigenvalues, edited by Olga Taussky, Nat. Bur. Standards. Appl. Math. Ser., v. 39, 1954, p. $117-130$.

2. David G. Feingold \& Richard S. VarGa, "Block diagonally dominant matrices and generalizations of the Gerschgorin Circle Theorem," Case Institute of Technology, Cleveland 6, Ohio, Report No. 1062, 1962.

3. A. S. Housenolder, "The approximate solution of matrix problems," J. Assoc. Comput. Mach., v. 5, 1958, p. 205-243.

\section{Supplementary Data and Remarks Concerning a Hardy-Littlewood Conjecture}

\section{By Daniel Shanks}

Let $P_{a}(N)$ be the number of primes of the form $n^{2}+a$ for $1 \leqq n \leqq N$, and let $\bar{\pi}_{a}(N)$ be the number of primes $\leqq N$ for which $-a$ is a quadratic nonresidue. In [1] we discussed a conjecture of Hardy and Littlewood to the effect that

$$
\frac{P_{a}(N)}{\bar{\pi}_{a}(N)} \sim h_{a}
$$

where the constant $h_{a}$ is given by

$$
h_{a}=\prod_{p \nmid a}\left(1-\left(\frac{-a}{p}\right) \frac{1}{p-1}\right),
$$

the product being taken over the odd primes $p$, with $(-a / p)$ the Legendre Symbol. We gave in [1] a heuristic argument in support of (1), a method of computing the $h_{a}$, and supporting empirical data for the six cases $a=1, \pm 2, \pm 3$, and 4 .

Subsequently the primes were also counted for six other cases, namely $a=$ $\pm 5, \pm 6, \pm 7$, and since such data are not available elsewhere it seems desirable to record them in a brief note. In Tables 1,2 , and 3 we show summaries for $N=$ $10,000(10,000) 180,000$ in the same format as the tables in [1].

While accurate values of $h_{a}$ in these six cases had not been computed, it was at once apparent that (1) is at least roughly correct for these values of $a$ also. Quite recently [2] tables of $L_{a}(s)$ for $a= \pm 6$ have been computed by J. W. Wrench, Jr., and, on the basis of these, one finds

$$
\begin{aligned}
h_{6} & =0.71304162 \\
h_{-6} & =1.03575587 .
\end{aligned}
$$

These are in good agreement with the empirical ratios in Table 2. Equally accurate constants for $a= \pm 5$ and \pm 7 are more difficult to compute, and are not yet available.

We may note the following:

1. Of the twelve forms, $n^{2}+a$, that we have investigated, $n^{2}+7$ has the most primes. Its (empirical) $h_{7}$, equal to 1.98 , indicates that numbers of this form are primes nearly twice as often as numbers of the same magnitude chosen at random.

Received November 8, 1962. 